PROCEEDINGS OF THE

AMERICAN MATHEMATICAL SOCIETY

Volume 135, Number 1, January 2007, Pages 289-298

S 0002-9939(06)08510-8

Article electronically published on July 28, 2006

\title{
A UNIQUENESS RESULT OF KÄHLER RICCI FLOW WITH AN APPLICATION
}

\author{
XU-QIAN FAN
}

(Communicated by Richard A. Wentworth)

\begin{abstract}
In this paper, we will study the problem of uniqueness of Kähler Ricci flow on some complete noncompact Kähler manifolds and the convergence of the flow on $\mathbb{C}^{n}$ with the initial metric constructed by Wu and Zheng.
\end{abstract}

\section{INTRODUCTION}

It is well known that the Ricci flow was initially introduced by Hamilton [7, 8, and that the short time existence and uniqueness in the compact case were proved therein. Letting $\left(M^{n}, g_{\alpha \bar{\beta}}(x)\right)$ be a complete noncompact Kähler manifold, we will consider the Kähler Ricci flow

$$
\left\{\begin{array}{l}
\frac{\partial}{\partial t} g_{\alpha \bar{\beta}}(x, t)=-R_{\alpha \bar{\beta}}(x, t) \\
g_{\alpha \bar{\beta}}(x, 0)=g_{\alpha \bar{\beta}}(x)
\end{array}\right.
$$

where $R_{\alpha \bar{\beta}}(x, t)$ is the Ricci curvature with respect to $g_{\alpha \bar{\beta}}(x, t)$. W.-X. Shi [13, 14 ] proved the following short time existence for the above system. See Theorem 1.1 in [13] and Theorems 2.1 and 5.1 in [14.

Theorem 1.1. Let $\left(M^{n}, g_{\alpha \bar{\beta}}(x)\right)$ be a complete noncompact Kähler manifold with Riemannian curvature tensor bounded by $K_{0}$. Then (1.1) has a solution $g_{\alpha \bar{\beta}}(x, t)$ on $M \times[0, T]$ for some $T\left(n, K_{0}\right)$ which is a family of Kähler metrics on $M$ satisfying

$$
C^{-1} g_{\alpha \bar{\beta}}(x) \leq g_{\alpha \bar{\beta}}(x, t) \leq C g_{\alpha \bar{\beta}}(x)
$$

for all $(x, t) \in M \times[0, T]$, where $C$ is a constant depending only on $n, K_{0}$ and $T$.

We want to apply a maximum principle to show that the above solution is unique if the Ricci tensor has a potential with respect to the initial metric. More precisely, we have the following:

Theorem 1.2. Let $\left(M^{n}, g_{\alpha \bar{\beta}}(x)\right)$ be a complete noncompact Kähler manifold with bounded Riemannian curvature tensor. Suppose there is a smooth function $f(x)$ on $M$ such that $\sqrt{-1} \partial \bar{\partial} f=$ Ric, where Ric is the Ricci form of the metric $g_{\alpha \bar{\beta}}(x)$.

Received by the editors July 5, 2005 and, in revised form, August 10, 2005.

2000 Mathematics Subject Classification. Primary 53C44; Secondary 58J37, 35B35.

Key words and phrases. Kähler Ricci flow.

This research was partially supported by the studentship of The Chinese University of Hong Kong and the Foundation of Jinan University.

(C)2006 American Mathematical Society Reverts to public domain 28 years from publication 
Suppose $g_{\alpha \bar{\beta}}(x, t)$ and $\tilde{g}_{\alpha \bar{\beta}}(x, t)$ are two solutions on $M \times[0, T]$ to (1.1) with the same initial metric $g_{\alpha \bar{\beta}}(x)$ satisfying

$$
c^{-1} g_{\alpha \bar{\beta}}(x) \leq g_{\alpha \bar{\beta}}(x, t), \quad \tilde{g}_{\alpha \bar{\beta}}(x, t) \leq c g_{\alpha \bar{\beta}}(x)
$$

for some constant $c>0$ such that $g_{\alpha \bar{\beta}}(x, t)$ and $\tilde{g}_{\alpha \bar{\beta}}(x, t)$ are Kähler for all $t \in[0, T]$. Then $g_{\alpha \bar{\beta}}(x, t)=\tilde{g}_{\alpha \bar{\beta}}(x, t)$ on $M \times[0, T]$.

Recently, Chen and Zhu [5] proved independently the uniqueness result for the Ricci flow up to some time $T$ assuming only the curvature is bounded at each time $t \in[0, T]$.

This paper is organized as follows. In Section 2, we will prove Theorem 1.2. In Section 3, we will apply the result to study the convergence of Kähler Ricci flows on $\mathbb{C}^{n}$ with the initial metrics constructed by Wu and Zheng [16.

\section{The Proof of Theorem 1.2}

We need the following result about exhaustion functions, which is due to W.-X. Shi; see Theorem 3.6 in [14.

Lemma 2.1. Suppose $\left(M^{n}, g_{i j}(x)\right)$ is an n-dimensional complete noncompact Riemannian manifold and its Riemannian curvature tensor $\left\{R_{i j k l}\right\}$ satisfies

$$
\left|R_{i j k l}\right|^{2} \leq k_{0}
$$

on $M$ for some constant $k_{0}$. Then there is a constant $C_{1}\left(n, k_{0}\right)$ such that for any fixed point $x_{0} \in M$, there is a smooth function $\psi(x) \in C^{\infty}(M)$ satisfying

$$
\left\{\begin{array}{l}
C_{1}^{-1}\left[1+r\left(x, x_{0}\right)\right] \leq \psi(x) \leq C_{1}\left[1+r\left(x, x_{0}\right)\right] \\
|\nabla \psi(x)| \leq C_{1} \\
\left|\nabla_{i} \nabla_{j} \psi(x)\right| \leq C_{1}
\end{array}\right.
$$

for all $x \in M$.

Now we will prove Theorem 1.2 .

Proof of Theorem 1.2. For any fixed point $x \in M$, let

$$
u(x, t)=\int_{0}^{t}\left(\log \frac{\operatorname{det}\left(g_{\alpha \bar{\beta}}(x, \tau)\right)}{\operatorname{det}\left(g_{\alpha \bar{\beta}}(x)\right)}-f(x)\right) d \tau
$$

for all $t \in[0, T]$. One has

$$
\begin{cases}\frac{\partial}{\partial t} u(x, t) & =\log \frac{\operatorname{det}\left(g_{\alpha \bar{\beta}}(x, t)\right)}{\operatorname{det}\left(g_{\alpha \bar{\beta}}(x)\right)}-f(x), \\ u(x, 0) & =0 .\end{cases}
$$

Noting that $R_{\alpha \bar{\beta}}(x)=f_{\alpha \bar{\beta}}(x)$ and $R_{\alpha \bar{\beta}}(x, t)=-\left(\log \operatorname{det}\left(g_{i \bar{j}}(x, t)\right)\right)_{\alpha \bar{\beta}}$, one has

$$
\begin{aligned}
\left(u_{t}\right)_{\alpha \bar{\beta}}(x, t) & =-R_{\alpha \bar{\beta}}(x, t)+R_{\alpha \bar{\beta}}(x)-f_{\alpha \bar{\beta}}(x) \\
& =-R_{\alpha \bar{\beta}}(x, t) .
\end{aligned}
$$

As in [2], let

$$
S_{\alpha \bar{\beta}}(x, t)=g_{\alpha \bar{\beta}}(x, t)-g_{\alpha \bar{\beta}}(x)-u_{\alpha \bar{\beta}}(x, t) .
$$

From $\frac{\partial}{\partial t} g_{\alpha \bar{\beta}}(x, t)=-R_{\alpha \bar{\beta}}(x, t)$, one has

$$
\frac{\partial}{\partial t} S_{\alpha \bar{\beta}}(x, t)=\frac{\partial}{\partial t} g_{\alpha \bar{\beta}}(x, t)-\frac{\partial}{\partial t} u_{\alpha \bar{\beta}}(x, t)=0 .
$$


So

$$
\frac{\partial}{\partial t} S_{\alpha \bar{\beta}}(x, t)=0 \text { with } S_{\alpha \bar{\beta}}(x, 0)=0 .
$$

Hence $S_{\alpha \bar{\beta}}(x, t)=0$. That is, $g_{\alpha \bar{\beta}}(x, t)=g_{\alpha \bar{\beta}}(x)+u_{\alpha \bar{\beta}}(x, t)$.

Similarly, letting

$$
v(x, t)=\int_{0}^{t}\left(\log \frac{\operatorname{det}\left(\tilde{g}_{\alpha \bar{\beta}}(x, \tau)\right)}{\operatorname{det}\left(g_{\alpha \bar{\beta}}(x)\right)}-f(x)\right) d \tau
$$

one has

$$
\begin{cases}\frac{\partial}{\partial t} v(x, t) & =\log \frac{\operatorname{det}\left(\tilde{g}_{\alpha \bar{\beta}}(x, t)\right)}{\operatorname{det}\left(g_{\alpha \bar{\beta}}(x)\right)}-f(x), \\ v(x, 0) & =0\end{cases}
$$

with $\tilde{g}_{\alpha \bar{\beta}}(x, t)=g_{\alpha \bar{\beta}}(x)+v_{\alpha \bar{\beta}}(x, t)$. Setting $w=u-v$, one has

$$
w(x, t)=\int_{0}^{t}\left(\log \frac{\operatorname{det}\left(g_{\alpha \bar{\beta}}(x, \tau)\right)}{\operatorname{det}\left(\tilde{g}_{\alpha \bar{\beta}}(x, \tau)\right)}\right) d \tau \leq 2 n T \log c
$$

for all $(x, t) \in M \times[0, T]$, where $c$ is the same as in (1.3), and

$$
\begin{cases}\frac{\partial}{\partial t} w(x, t) & =\log \frac{\operatorname{det}\left(g_{\alpha \bar{\beta}}(x)+u_{\alpha \bar{\beta}}(x, t)\right)}{\operatorname{det}\left(g_{\alpha \bar{\beta}}(x)+v_{\alpha \bar{\beta}}(x, t)\right)}, \\ w(x, 0) & =0 .\end{cases}
$$

As in [3] (see the proof of Lemma 6.2 in [3]), one has

$$
\begin{aligned}
& \log \frac{\operatorname{det}\left(g_{\alpha \bar{\beta}}(x)+u_{\alpha \bar{\beta}}(x, t)\right)}{\operatorname{det}\left(g_{\alpha \bar{\beta}}(x)+v_{\alpha \bar{\beta}}(x, t)\right)} \\
= & \log \operatorname{det}\left(g_{\alpha \bar{\beta}}(x)+u_{\alpha \bar{\beta}}(x, t)\right)-\log \operatorname{det}\left(g_{\alpha \bar{\beta}}(x)+v_{\alpha \bar{\beta}}(x, t)\right) \\
= & \int_{0}^{1} \frac{\partial}{\partial s} \log \operatorname{det}\left(g_{\alpha \bar{\beta}}(x)+s u_{\alpha \bar{\beta}}(x, t)+(1-s) v_{\alpha \bar{\beta}}(x, t)\right) d s \\
= & \int_{0}^{1} \triangle_{(t, s)} w d s,
\end{aligned}
$$

where $\triangle_{(t, s)}$ is the Laplacian operator with respect to the Kähler metric

$$
h_{\alpha \bar{\beta}}(x, t, s)=g_{\alpha \bar{\beta}}(x, t)+s u_{\alpha \bar{\beta}}(x, t)+(1-s) v_{\alpha \bar{\beta}}(x, t)
$$

on $M \times[0, T] \times[0,1]$. That is, $\triangle_{(t, s)} w(x, t)=h^{\alpha \bar{\beta}}(x, t, s) w_{\alpha \bar{\beta}}(x, t)$. So

$$
\frac{\partial}{\partial t} w(x, t)-\int_{0}^{1} \triangle_{(t, s)} w d s=0
$$

for all $(x, t, s) \in M \times[0, T] \times[0,1]$. From (1.3), one has for any $s \in[0,1]$,

$$
c^{-1} g_{\alpha \bar{\beta}}(x) \leq s g_{\alpha \bar{\beta}}(x, t)+(1-s) \tilde{g}_{\alpha \bar{\beta}}(x, t) \leq c g_{\alpha \bar{\beta}}(x)
$$

for all $(x, t) \in M \times[0, T] \times[0,1]$. That is equivalent to

$$
c^{-1} g_{\alpha \bar{\beta}}(x) \leq h_{\alpha \bar{\beta}}(x, t, s) \leq c g_{\alpha \bar{\beta}}(x)
$$

for all $(x, t, s) \in M \times[0, T] \times[0,1]$. Since the Riemannian curvature tensor with respect to $g_{\alpha \bar{\beta}}(x)$ is bounded, by Lemma 2.1, there is a real function $\psi(x) \in C^{\infty}(M)$ such that

$$
\left\{\begin{array}{l}
\mu^{-1}(1+r(x)) \leq \psi(x) \leq \mu(1+r(x)) \\
|\nabla \psi| \leq \mu \\
\left|\nabla_{i} \nabla_{j} \psi\right| \leq \mu
\end{array}\right.
$$


for some constant $\mu>1$, where $\nabla \psi$ and $\nabla_{i} \nabla_{j} \psi$ are the gradient and Hessian of $\psi$ with respect to $g_{\alpha \bar{\beta}}(x)$. Since $\psi_{\alpha \bar{\beta}}$ is a Hermitian symmetric $(1,1)$ tensor, by the last inequality of (2.11), one has that at any point $x \in M$

$$
\left(\inf _{0 \neq \nu \in T_{x}^{1,0} M} \frac{\psi_{\alpha \bar{\beta}}(x)(\nu, \bar{\nu})}{g_{\alpha \bar{\beta}}(x)(\nu, \bar{\nu})}\right)^{2}+\left(\sup _{0 \neq \nu \in T_{x}^{1,0} M} \frac{\psi_{\alpha \bar{\beta}}(x)(\nu, \bar{\nu})}{g_{\alpha \bar{\beta}}(x)(\nu, \bar{\nu})}\right)^{2} \leq C(n) \mu^{2}
$$

for some constant $C(n)$ depending only on $n$. By (2.10), one has for any $0 \neq \nu \in$ $T_{x}^{1,0} M$

$$
\begin{aligned}
\left|\frac{\psi_{\alpha \bar{\beta}}(x)(\nu, \bar{\nu})}{h_{\alpha \bar{\beta}}(x, t, s)(\nu, \bar{\nu})}\right| & =\left|\frac{\psi_{\alpha \bar{\beta}}(x)(\nu, \bar{\nu})}{g_{\alpha \bar{\beta}}(x)(\nu, \bar{\nu})}\right| \cdot\left|\frac{g_{\alpha \bar{\beta}}(x)(\nu, \bar{\nu})}{h_{\alpha \bar{\beta}}(x, t, s)(\nu, \bar{\nu})}\right| \\
& \leq c\left|\frac{\psi_{\alpha \bar{\beta}}(x)(\nu, \bar{\nu})}{g_{\alpha \bar{\beta}}(x)(\nu, \bar{\nu})}\right|,
\end{aligned}
$$

where $c$ is the same as in (2.10). So there is another constant $C(c, n, \mu)$ such that

$$
\left\{\begin{array}{l}
C^{-1}\left(1+r^{(t, s)}(x)\right) \leq \psi(x) \leq C\left(1+r^{(t, s)}(x)\right) \\
\left|\nabla^{(t, s)} \psi\right|_{(t, s)} \leq C, \\
\left|\triangle_{(t, s)} \psi\right| \leq C
\end{array}\right.
$$

where $r^{(t, s)}(x),\left|\nabla^{(t, s)} \psi\right|_{(t, s)}$ and $\triangle_{(t, s)} \psi$ are taken with respect to $h_{\alpha \bar{\beta}}(x, t, s)$ for all $(t, s) \in[0, T] \times[0,1]$. Let $W(x, t)=e^{\lambda t} \psi(x)$, where $\lambda$ will be chosen later. We have

$$
W_{t}=\lambda e^{\lambda t} \psi(x) \text { and } \triangle_{(t, s)} W(x, t)=e^{\lambda t} \triangle_{(t, s)} \psi
$$

and (2.13) implies that

$$
\triangle_{(t, s)} W(x, t) \leq C e^{\lambda t}
$$

where $C$ is the constant in (2.13). Setting $\lambda=C^{2}+1$ for example, one has

$$
W_{t}-\triangle_{(t, s)} W(x, t)>0
$$

for all $(x, t, s) \in M \times[0, T] \times[0,1]$. For any $\epsilon>0$, setting

$$
\theta_{\epsilon}(x, t)=w(x, t)-\epsilon W(x, t),
$$

one has $\theta_{\epsilon}(x, t)<0$ for $x$ tending to infinity because $w$ is bounded for all $(x, t) \in$ $M \times[0, T]$ by (2.7). Hence if $\theta_{\epsilon}(x, t)>0$ somewhere, then $\theta_{\epsilon}(x, t)$ has a maximal value at some point $\left(x_{1}, t_{1}\right) \in M \times[0, T]$. By the maximum principle, at $\left(x_{1}, t_{1}\right)$,

$$
0 \leq\left(\frac{\partial}{\partial t}-\triangle_{(t, s)}\right) \theta_{\epsilon}(x, t) .
$$

So by (2.9) and (2.14), one has

$$
\begin{aligned}
0 & \leq \frac{\partial}{\partial t} \theta_{\epsilon}(x, t)-\int_{0}^{1} \triangle_{(t, s)} \theta_{\epsilon}(x, t) d s \\
& =-\epsilon\left(W_{t}-\int_{0}^{1} \triangle_{(t, s)} W(x, t) d s\right) \\
& <0 .
\end{aligned}
$$

This is a contradiction. Hence $w(x, t) \leq \epsilon W(x, t)$. Letting $\epsilon \rightarrow 0$, we have $w(x, t) \leq$ 0 , i.e., $u \leq v$. By symmetry, we have $u(x, t)=v(x, t)$. Therefore $g_{\alpha \bar{\beta}}(x, t)=$ $\tilde{g}_{\alpha \bar{\beta}}(x, t)$. 
The existence of the potential to the Ricci tensor is related to solving the corresponding Poincaré-Lelong equation $\sqrt{-1} \partial \bar{\partial} u=$ Ric. This question was extensively studied (see [9, 11, 10, 15, 6]). In particular, from Theorem 6.1 in 11] (see also Theorem 5.1 in [10]) and Theorem 3.3 in [6], one has that if $\left(M^{n}, g_{\alpha \bar{\beta}}(x)\right)$ is a complete noncompact Kähler manifold of complex dimension $n$ with nonnegative holomorphic bisectional curvature and bounded scalar curvature $\mathcal{R}$, then the Poincaré-Lelong equation has a solution, provided either

(i) $\int_{0}^{\infty} f_{B_{o}(t)} \mathcal{R} d t<\infty$, or

(ii) the Ricci curvature $\operatorname{Ricci}(x) \geq \frac{a \ln \ln (10+r(x))}{\left(1+r^{2}(x)\right) \ln (10+r(x))}$ for some $a>268(n+2)^{2}$ and all group homomorphisms from $\pi_{1}(M)$ to $\mathbb{R}$ are trivial; moreover the universal covering space $\widetilde{M}$ of $M$ with the covering metric has no compact factors.

\section{Convergence of some KäHler Ricci flows}

As a consequence of Theorem 1.2, we will show that the rotational symmetry is preserved under the Kähler-Ricci flow under a certain assumption. More precisely, we have

Proposition 3.1. Let $g_{\alpha \bar{\beta}}(x)$ be a complete Kähler metric on $\mathbb{C}^{n}$ which is rotationally symmetric, i.e., $U(n)$-invariant, satisfying that the Riemannian curvature tensor with respect to $g_{\alpha \bar{\beta}}(x)$ is bounded. Suppose a family of Kähler metrics $g_{\alpha \bar{\beta}}(x, t)$ on $\mathbb{C}^{n}$ for $t \in[0, T]$ is a solution to (1.1) with the initial value $g_{\alpha \bar{\beta}}(x)$ such that (1.2) holds for some constant $C$ and for all $(x, t) \in \mathbb{C}^{n} \times[0, T]$. Then $g_{\alpha \bar{\beta}}(x, t)$ is also rotationally symmetric for all $t \in[0, T]$.

Proof. Let $\left(z^{1}, \cdots, z^{n}\right)$ be the standard coordinate on $\mathbb{C}^{n}$. Then

$$
g_{\alpha \bar{\beta}}(z, t)=\left\langle\frac{\partial}{\partial z^{\alpha}}(z), \frac{\partial}{\partial \bar{z}^{\beta}}(z)\right\rangle_{t}
$$

For any $\phi \in U(n)$, setting $\left(y^{1}, \cdots, y^{n}\right)=\phi^{-1}\left(z^{1}, \cdots, z^{n}\right)$ and $\tilde{g}=\phi^{*} g$, which is the pulled-back of $g$, one has

$$
\tilde{R}_{\alpha \bar{\beta}}(y, t)=\phi^{*} \operatorname{Ricci}\left(\frac{\partial}{\partial y^{\alpha}}(y), \frac{\partial}{\partial \bar{y}^{\beta}}(y)\right),
$$

where $\tilde{R}_{\alpha \bar{\beta}}(y, t)$ is the Ricci curvature with respect to $\tilde{g}_{\alpha \bar{\beta}}(y, t)$. Since $\frac{\partial}{\partial t} g_{\alpha \bar{\beta}}=$ $-R_{\alpha \bar{\beta}}$, one has $\frac{\partial}{\partial t} \tilde{g}_{\alpha \bar{\beta}}=-\tilde{R}_{\alpha \bar{\beta}}$. Since the initial metric $g$ is rotationally symmetric, i.e., $g=\phi^{*} g$, one has that there is a function $\varphi(z, \bar{z})$ such that $g_{\alpha \bar{\beta}}=\varphi_{\alpha \bar{\beta}}$, where $z \in \mathbb{C}^{n}$, and $\varphi(z, \bar{z})=w\left(|z|^{2}\right)$ for some smooth function $w$. See, for example, page 4 in [1]. So $-\log \frac{\operatorname{det}\left(g_{\alpha \bar{\beta}}\right)}{\operatorname{det}\left(\delta_{\alpha \bar{\beta}}\right)}$ is a potential of Ricci tensor, where $\delta_{\alpha \bar{\beta}}$ is the standard metric on $\mathbb{C}^{n}$. Since $g_{\alpha \bar{\beta}}(x, t)$ is uniformly equivalent to $g_{\alpha \bar{\beta}}(x)$ and $g_{\alpha \bar{\beta}}(x)$ is $U(n)$-invariant, one has that (1.2) is also true for $\tilde{g}$. By Theorem [1.2, we have $g(t)=\phi^{*} g(t)$. That is, the solution $g(x, t)$ is rotationally symmetric.

From now until the end of the paper, we will study the Kähler Ricci flow with the initial metric on $\mathbb{C}^{n}$ constructed by Wu and Zheng [16] and $g_{i \bar{j}}(z ; c)$ will always denote the metric that will be defined in (3.1).

For convenience, let us recall some part of the results in [16]. See Example 2 in [16]. Let $z=\left(z^{1}, \cdots, z^{n}\right)$ be the standard coordinate on $\mathbb{C}^{n}$, and

$$
f=\frac{R^{1-c}-1}{(1-c) r},
$$


where $R=1+r, r(z)=|z|^{2}$ and $0<c<1$. Then for any fixed $0<c<1$, the Kähler metric

$$
g_{i \bar{j}}(z ; c)=f(r) \delta_{i j}+f^{\prime}(r) \bar{z}_{i} z_{j}
$$

on $\mathbb{C}^{n}$ is complete with positive bisectional curvature. Moreover it has maximal volume growth and quadratic curvature decay. The scalar curvature function $\mathcal{R}(z)$ is given by

$$
\mathcal{R}(z)=A+2(n-1) B+\frac{1}{2} n(n-1) C,
$$

where

$$
A=\frac{c}{R^{2-c}}, B=\frac{1}{f^{2} R r}(1-f), C=\frac{2}{f^{2} r}\left(f-R^{-c}\right) .
$$

The distance function to the origin is given by

$$
s(z)=\int_{0}^{r} \frac{1}{2 \sqrt{\tau(1+\tau)^{c}}} d \tau .
$$

Let $B_{0}(s)$ be the geodesic ball centered at the origin with radius $s$ with respect to the metric $g_{i \bar{j}}(z ; c)$, i.e., with radius $\sqrt{r}$ with respect to the standard metric. Then the volume of it with respect to $g_{i \bar{j}}(z ; c)$ is given by $V_{o}(s)=\omega_{n}(r f)^{n}$, where $\omega_{n}$ is the volume of unit ball in $\mathbb{C}^{n}$ with respect to the standard metric. In [17, Zheng pointed out $\mathcal{R s}^{2}(z) \leq c Q$ for $0<c<1 / 2$, where $Q$ is a constant depending only on $n, V_{o}(s) /\left(\omega_{n} s^{2 n}\right) \geq(1-c)^{n}$ for $0<c<1$, with a sketch of the proof for these facts. For convenience, we will modify slightly the statement and explain his idea in greater detail.

Lemma 3.2. With the above notation, $V_{o}(s) \geq \omega_{n} s^{2 n}(1-c)^{n}$ for $0<c<1$, and $\mathcal{R} R^{1-c} \leq\left(2 n^{2}-n\right) c$ for $0<c \leq 1 / 2$.

Proof. We will check the volume growth first. Setting $u(r)=\frac{r f}{s^{2}}$, one has

$$
u^{\prime}=s^{-3}(1-c)^{-1} R^{-c} u_{1},
$$

where

$$
u_{1}=s(1-c)-r^{-1 / 2} R^{1-\frac{c}{2}}+r^{-1 / 2} R^{c / 2} .
$$

We want to show $u_{1} \leq 0$. First $u_{1}(0)=\lim _{r \rightarrow 0} \frac{R^{c / 2}-R^{1-c / 2}}{r^{1 / 2}}=0$,

$$
u_{1}^{\prime}=r^{-\frac{3}{2}} u_{2},
$$

where $u_{2}=\frac{1}{2} R^{-\frac{c}{2}}+\left(-\frac{1}{2}+\frac{c}{2}\right) R^{\frac{c}{2}}-\frac{c}{2} R^{\frac{c}{2}-1}$. Noting that $u_{2}(0)=0$ and

$$
\begin{aligned}
u_{2}^{\prime} & =c R^{-\frac{c}{2}-2}\left[-\frac{1}{4} R+\left(-\frac{1}{4}+\frac{c}{4}\right) R^{1+\frac{3 c}{2}}-\left(\frac{c}{4}-\frac{1}{2}\right) R^{c}\right] \\
& =c R^{-\frac{c}{2}-2} u_{3},
\end{aligned}
$$

while $u_{3}(0)=0$ and $u_{3}^{\prime}(0)<0$ with $u_{3}^{\prime \prime}<0$, one can get $u_{1} \leq 0$ and $u$ is nonincreasing. By (3.3), one has $s \leq \frac{1}{2} \int_{0}^{r} \tau^{\frac{-1-c}{2}} d \tau=\frac{1}{1-c} r^{\frac{1-c}{2}}$ as $0<c<1$. So $u \geq \lim _{r \rightarrow \infty} \frac{r f}{s^{2}} \geq 1-c$. Now we will check the curvature decay. Clearly $A \times R^{1-c} \leq c$. Since $R^{-c} \leq f$, one has

$$
\begin{aligned}
B \times R^{1-c} & =f^{-2} R^{-2 c} R^{c} r^{-1}(1-f) \\
& \leq R^{c} r^{-1}(1-f) .
\end{aligned}
$$


We want to show that $R^{c} r^{-1}(1-f)$ is nonincreasing in $r \in(0, \infty)$ as $0<c \leq 1 / 2$. Letting $B_{1}=R^{c} r^{-1}(1-f)=\frac{(1-c) r R^{c}-R+R^{c}}{(1-c) r^{2}}$, one has

$$
B_{1}^{\prime}=\frac{-(1-c) R^{c} r+c(1-c) r^{2} R^{c-1}-r+c R^{c-1} r+2 R-2 R^{c}}{(1-c) r^{3}}=\frac{B_{2}}{(1-c) r^{3}}
$$

with $B_{2}(0)=0$;

$$
B_{2}^{\prime}=\left(-1+c+c^{2}-c^{3}\right) R^{c}+\left(-c-2 c^{2}+2 c^{3}\right) R^{c-1}+\left(c^{2}-c^{3}\right) R^{c-2}+1
$$

with $B_{2}^{\prime}(0)=0$;

$$
\begin{aligned}
B_{2}^{\prime \prime} & =R^{c-3}\left[\left(-c+c^{2}+c^{3}-c^{4}\right) R^{2}+\left(c+c^{2}-4 c^{3}+2 c^{4}\right) R+\left(-2 c^{2}+3 c^{3}-c^{4}\right)\right] \\
& =R^{c-3} B_{3}
\end{aligned}
$$

with $B_{3}(0)=0$;

$$
B_{3}^{\prime}=2\left(-c+c^{2}+c^{3}-c^{4}\right) R+\left(c+c^{2}-4 c^{3}+2 c^{4}\right)
$$

with $B_{3}^{\prime}(0)=-c\left(1-3 c+2 c^{2}\right) \leq 0$ for $0 \leq c \leq 1 / 2$; and

$$
B_{3}^{\prime \prime}=-2 c\left(1-c-c^{2}+c^{3}\right) \leq 0 .
$$

So $2(n-1) B R^{1-c} \leq(n-1) c$, because $B_{1}(0)=\lim _{r \rightarrow 0} \frac{(1-c) r-R^{1-c}+1}{(1-c) r^{2}}=\frac{c}{2}$. Clearly

$$
\begin{aligned}
\frac{1}{2} n(n-1) C R^{1-c} & =n(n-1) f^{-2} R^{-2 c} r^{-1}\left(f-R^{-c}\right) R^{1+c} \\
& \leq n(n-1) r^{-1} R^{1+c}\left(f-R^{-c}\right) .
\end{aligned}
$$

Letting $C_{1}=r^{-1} R^{1+c}\left(f-R^{-c}\right)$, one has

$$
C_{1}^{\prime}=\frac{(1-c) R^{1+c}-(1+c) R+(1+c) R^{c}-(1-c)}{(1-c) r^{3}}=\frac{C_{2}}{(1-c) r^{3}}
$$

with $C_{2}(0)=0$, and

$$
C_{2}^{\prime}=\left(1-c^{2}\right) R^{c}-(1+c)+c(1+c) R^{c-1}
$$

with $C_{2}^{\prime}(0)=0$, while

$$
C_{2}^{\prime \prime}=c\left(1-c^{2}\right) R^{c-1}-c\left(1-c^{2}\right) R^{c-2} \geq 0 .
$$

So $C_{1}$ is nondecreasing in $r$. Since $C_{1}(\infty)=\frac{c}{1-c}$, we have

$$
\frac{1}{2} n(n-1) C R^{1-c} \leq n(n-1) \frac{c}{1-c} .
$$

By (3.2), (3.4) and (3.5), one can get the second part of the lemma.

We will claim that the averages of the scalar curvature on the geodesic balls on any fixed center are uniformly quadratic decay in the radii of the geodesic balls as $n \geq 2$. Since $(1-c) s^{2} \leq r f=\frac{R^{1-c}-1}{1-c}$, one has

$$
\frac{(1-c)^{2}}{R^{1-c}} \leq \frac{1}{1+s^{2}}
$$

for $0<c<1$, and then

$$
\mathcal{R} \leq \frac{c_{1}}{1+s^{2}}
$$


for $0<c \leq 1 / 2$, where $c_{1}=\frac{\left(2 n^{2}-n\right) c}{(1-c)^{2}}$. Since $V_{o}(s) \geq \omega_{n} s^{2 n}(1-c)^{n}$, by volume comparison, one has, for $n \geq 2$,

$$
\begin{aligned}
\frac{1}{V_{o}(s)} \int_{B_{o}(s)} \mathcal{R} & =\frac{1}{V_{o}(s)} \int_{0}^{s}\left(\int_{\partial B_{o}(t)} \mathcal{R}\right) d t \\
& \leq \frac{1}{V_{o}(s)} \int_{0}^{s} \int_{\partial B_{o}(t)} \frac{c_{1}}{1+t^{2}} d t \\
& \leq \frac{c_{1}}{1+s^{2}}+\frac{2 c_{1}}{s^{2 n}(1-c)^{n}} \int_{0}^{s} \frac{t^{2 n+1}}{\left(1+t^{2}\right)^{2}} d t \\
& \leq \frac{c_{2}}{1+s^{2}},
\end{aligned}
$$

where $c_{2}=\left(1+\frac{1}{(n-1)(1-c)^{n}}\right) c_{1}$. Now we will show that, for $n \geq 2$,

$$
\frac{1}{V_{x}(s)} \int_{B_{x}(s)} \mathcal{R} \leq \frac{\tilde{c}}{1+s^{2}}
$$

for $0<c \leq 1 / 2, x \in \mathbb{C}^{n}$, where $\tilde{c}=\frac{c_{2} 3^{2 n}}{(1-c)^{n}}$. For $s \geq r(x) / 2$, by Lemma 3.2 and (3.6), one has

$$
\begin{aligned}
\frac{1}{V_{x}(s)} \int_{B_{x}(s)} \mathcal{R} & \leq \frac{V_{o}(s+r)}{V_{x}(s)} \cdot \frac{1}{V_{o}(s+r)} \int_{B_{o}(s+r)} \mathcal{R} \\
& \leq \frac{\omega_{n}(s+r)^{2 n}}{\omega_{n} s^{2 n}(1-c)^{n}} \cdot \frac{1}{V_{o}(s+r)} \int_{B_{o}(s+r)} \mathcal{R} \\
& \leq \frac{\tilde{c}}{1+s^{2}} .
\end{aligned}
$$

For $s<r(x) / 2$, it is easy to see that (3.7) holds.

In [12, Ni and Tam proved the following result (see Theorem 1.3 in [12]).

Theorem 3.3. Let $\left(M^{n}, g_{\alpha \bar{\beta}}(x)\right)$ be a complete noncompact manifold with nonnegative holomorphic bisectional curvature such that its scalar curvature $\mathcal{R}_{0}$ is bounded and satisfies

$$
\int_{0}^{\infty} f_{B_{x}(s)} \mathcal{R}_{0} d s \leq C_{1}
$$

for some constant $C_{1}$ and for all $x$. Then (1.1) has a long-time solution $g_{\alpha \bar{\beta}}(x, t)$, which has nonnegative holomorphic bisectional curvature for any $t>0$. Moreover, there is a function $u(x, t)$ such that

$$
\left\{\begin{array}{l}
\sqrt{-1} \partial \bar{\partial} u(x, t)=\operatorname{Ric}\left(g_{\alpha \bar{\beta}}(x, t)\right) \\
|\nabla u| \leq C(n) C_{1} \\
\mathcal{R}(x, t)+|\nabla u|^{2}(x, t) \leq \sup _{M} \mathcal{R}_{0}+\left(C(n) C_{1}\right)^{2}
\end{array}\right.
$$

for all $(x, t)$.

So for any fixed $T>0, g_{\alpha \bar{\beta}}(x, t)$ is uniformly equivalent to $g_{\alpha \bar{\beta}}(x)$ for all $(x, t) \in$ $M^{n} \times[0, T]$. Hence by Proposition 3.1 (3.7) and Theorem 3.3, one has

Proposition 3.4. For any $0<c \leq 1 / 2$, the Kähler Ricci flow with the initial metric $g_{i \bar{j}}(z ; c)$ has a long-time solution $g_{i \bar{j}}(z, t ; c)$ which is rotationally symmetric for $t \geq$ 0 , and the scalar curvature has uniformly upper bound for all time $t$, moreover, the potential of the Ricci tensor with respect to $g_{i j}(z, t ; c)$ is of at most linear growth. 
The following theorem is due to Chau and Tam (see Theorem 1.1 or Theorem 4.3 in [4]).

Theorem 3.5. There exists a constant $\varsigma$ depending only on $n$ such that, if $M^{n}$ is a complete noncompact Kähler manifold with bounded nonnegative holomorphic bisectional curvature satisfying

(i)

$$
\frac{1}{V_{x}(r)} \int_{B_{x}(r)} \mathcal{R} \leq \frac{\varsigma}{1+r^{2}}
$$

for all $x \in M$ and for all $r>0$; and

(ii) there exist a point $p \in M$ and a sequence $t_{k} \rightarrow \infty$ such that $\frac{1}{\left|v_{p}\right|_{t_{k}}^{2}} g\left(p, t_{k}\right)$ are uniformly equivalent to $g(p, 0)$ at $p$, where $v_{p}$ is a fixed vector in $T_{p}^{1,0}(M)$ with $\left|v_{p}\right|_{0}=1$,

then the metrics $\frac{1}{\left|v_{p}\right|_{t_{k}}^{2}} g\left(x, t_{k}\right)$ subconverge uniformly in the $C^{\infty}$ topology in compact sets to a complete Kähler flat metric on $M$. In particular, the universal covering space of $M$ is biholomorphic to $\mathbb{C}^{n}$.

From Theorem 3.5, we have

Proposition 3.6. For $0<c \leq 1 / 2, n \geq 2$ and $\tilde{c} \leq \varsigma$, where $\tilde{c}, \varsigma$ are the same as in (3.7) and Theorem 3.5 respectively, there is a sequence $\left\{\mu_{k}\right\}$ such that $\mu_{k} g\left(z, t_{k} ; c\right)$ converges uniformly in the $C^{\infty}$ topology in compact sets to the standard metric on $\mathbb{C}^{n}$ up to a constant factor.

Proof. By Proposition 3.4 and Theorem 3.5, for $0<c \leq 1 / 2, n \geq 2$ and $\tilde{c} \leq \varsigma$, one has that there is a sequence $\left\{\mu_{k}\right\}$ such that $\mu_{k} g\left(z, t_{k} ; c\right)$ converges uniformly in the $C^{\infty}$ topology in compact sets to a complete Kähler flat metric on $\mathbb{C}^{n}$. Let $g(z ; c)=\lim _{k \rightarrow \infty} \mu_{k} g\left(z, t_{k} ; c\right)$. Since $\mu_{k} g\left(z, t_{k} ; c\right)$ is rotationally symmetric, it follows that $g(z ; c)$ will also be. So as in 1, for any chosen $c$, there is a smooth function $w(r ; c)$ on $[0, \infty)$ such that

$$
g_{i \bar{j}}(z ; c)=w_{i \bar{j}}\left(|z|^{2} ; c\right) .
$$

Letting $s=\log |z|^{2}$ and $u(s)=w\left(e^{s}\right)$, one has

$$
g_{i \bar{j}}(z ; c)=e^{-s} u^{\prime}(s) \delta_{i \bar{j}}+e^{-2 s} \bar{z}_{i} z_{j}\left(u^{\prime \prime}(s)-u^{\prime}(s)\right)
$$

and

$$
\operatorname{det}\left(g_{i \bar{j}}(z ; c)\right)=e^{-n s}\left(u^{\prime}(s)\right)^{n-1} u^{\prime \prime}(s) .
$$

Setting $\varrho(s)=-\log \operatorname{det}\left(g_{i \bar{j}}(z ; c)\right)$, one has

$$
R_{i \bar{j}}=\partial_{i} \partial_{\bar{j}} \varrho(s)=e^{-s} \varrho^{\prime}(s) \delta_{i \bar{j}}+e^{-2 s} \bar{z}_{i} z_{j}\left(\varrho^{\prime \prime}(s)-\varrho^{\prime}(s)\right) .
$$

So at point $z=\left(z_{1}, 0, \cdots, 0\right)$, one has

$$
R_{i \bar{j}}= \begin{cases}e^{-s} \varrho^{\prime \prime}(s), & i=i=1, \\ e^{-s} \varrho^{\prime}(s), & i=j \geq 2, \\ 0, & i \neq j .\end{cases}
$$

Since $g(z ; c)$ is flat, one has $e^{-s} \varrho^{\prime}(s)=e^{-s} \varrho^{\prime \prime}(s)=0$. So from (3.10),

$$
\varrho(s)=-\log \operatorname{det}\left(g_{i \bar{j}}(z ; c)\right)=\text { constant } n .
$$

This is equivalent to $e^{-n s}\left(u^{\prime}(s)\right)^{n-1} u^{\prime \prime}(s)=$ constant and then $\left(\left(u^{\prime}\right)^{n}\right)^{\prime}=n \lambda e^{n s}$ for some constant $\lambda>0$. So $\left(u^{\prime}(s)\right)^{n}=\lambda\left(e^{n s}-\lambda_{1}\right)$, where $\lambda_{1}$ is a constant. By (3.9), 
considering $i=j=2$, one has $g_{2 \overline{2}}(z ; c)=e^{-s} u^{\prime}(s)$. Since $g_{2 \overline{2}}(z ; c)$ is well defined at the origin, one has $\lim _{s \rightarrow-\infty} u^{\prime}(s)=0$. So $-\lambda \lambda_{1}=\lim _{s \rightarrow-\infty}\left(u^{\prime}(s)\right)^{n}=0$, and then $\lambda_{1}=0$. Hence $\left(u^{\prime}\right)^{n}=\lambda e^{n s}$, i.e., $u^{\prime}=\lambda^{1 / n} e^{s}$. Therefore $g_{i \bar{j}}(z ; c)=\lambda^{1 / n} \delta_{i \bar{j}}$. This completes the proof of the proposition.

\section{ACKNOWLEDGMENTS}

The main results of this paper were obtained when the author studied at The Chinese University of Hong Kong. He would like to thank Prof. Luen-Fai Tam for his inspired guidance, constant encouragement and very worthy advice. He would also like to thank the referee for pointing out a gap in Proposition 3.1, for giving many constructive suggestions that will make the presentation clearer, and for supplying the information on the paper [5].

\section{REFERENCES}

1. H.-D. Cao, Existence of gradient Kähler-Ricci solitons, Elliptic and parabolic methods in geometry (Minneapolis, MN, 1994), 1-16, A K Peters, Wellesley, MA, 1996. MR.1417944 (98a:53058)

2. A. Chau, Convergence of the Kähler-Ricci flow on noncompact manifolds, preprint.

3. A. Chau and O. C. Schnuerer, Stability of gradient Kähler-Ricci solitons, arXiv: math. DG/0307293.

4. A. Chau and L.-F. Tam, Gradient Kähler-Ricci solitons and a uniformization conjecture, arXiv: math.DG/0310198.

5. B.-L. Chen and X.-P. Zhu, Uniqueness of the Ricci flow on complete noncompact manifolds, arXiv: math.DG/0505447.

6. X.-Q. Fan, Thesis, The Chinese University of Hong Kong, (2004).

7. R. S. Hamilton, Three-manifolds with positive Ricci curvature, J. Differential Geom. 17 (1982), 255-306. MR0664497 (84a:53050)

8. R. S. Hamilton, The formation of singularities in the Ricci flow, Surv. Differ. Geom. 2 (1995), 7-136, International Press. MR1375255 (97e:53075)

9. N.-M. Mok, Y.-T. Siu and S.-T. Yau, The Poincaré-Lelong equation on complete Kähler manifolds, Compositio Math. 44 (1981), 183-218. MR0662462 (84g:32011)

10. L. Ni, Y.-G. Shi and L.-F. Tam, Poisson equation, Poincaré-Lelong equation and curvature decay on complete Kähler manifolds, J. Differential Geom. 57 (2001), 339-388. MR1879230 (2002j:53042)

11. L. Ni and L.-F. Tam, Plurisubharmonic functions and the structure of complete Kähler manifolds with nonnegative curvature, J. Differential Geom. 64 (2003), 457-524. MR2032112 (2005a:32023)

12. L. Ni and L.-F. Tam, Kähler-Ricci flow and the Poincare-Lelong equation, Comm. Anal. Geom. 12 (2004), 111-141. MR2074873 (2005f:53108)

13. W.-X. Shi, Deforming the metric on complete Riemannian manifolds, J. Differential Geom. 30 (1989), 223-301. MR1001277 (90i:58202)

14. W.-X. Shi, Ricci flow and the uniformization on complete noncompact Kähler manifolds, J. Differential Geom. 45 (1997), 94-220. MR 1443333 (98d:53099)

15. B. Wong and Q. Zhang, Refined gradient bounds, Poisson equations and some applications to open Kähler manifolds, Asian J. Math. 7 (2003), 337-364. MR2129327

16. H.-H. Wu and F.-Y. Zheng, Examples of positively curved complete Kähler manifolds, preprint.

17. F.-Y. Zheng, Private communications.

Department of Mathematics, Jinan University, Guangzhou, 510632 People's Republic OF CHINA

E-mail address: xqfan@hotmail.com 